\title{
Hairy root cultures of Gynostemma pentaphyllum (Thunb.) Makino: a promising approach for the production of gypenosides as an alternative of ginseng saponins
}

\author{
Chen-Kai Chang ${ }^{1}$, Ku-Shang Chang ${ }^{2}$, Yi-Chen Lin ${ }^{1}$, Sin-Yie Liu ${ }^{3} \&$ Chien-Yuan Chen ${ }^{1, *}$ \\ ${ }^{1}$ Institute of Microbiology and Biochemistry, National Taiwan University, Taipei, Taiwan \\ ${ }^{2}$ Department of Food Science, Yuanpei Institue of Science and Technology, Hsinchu, Taiwan \\ ${ }^{3}$ Department of Agronomy, Taiwan Agricultural Research Institute, Taichung, Taiwan \\ *Author for correspondence (Fax: +886-2-23661696; E-mail: chenyuan@ntu.edu.tw)
}

Received 24 March 2005; Revisions requested 14 April 2005; Revisions received 3 June 2005; Accepted 6 June 2005

Key words: Gynostemma pentaphyllum, gypenosides, hairy root cultures, jiaogulan

\begin{abstract}
Hairy root cultures of Gynostemma pentaphyllum were established by infecting leaf discs with Agrobacterium rhizogenes. The dry biomass of hairy roots grown in MS medium for 49 days was $7.3 \mathrm{~g} 1^{-1}$ with a gypenoside content of $38 \mathrm{mg} \mathrm{g}^{-1}$ dry wt.
\end{abstract}

\section{Introduction}

Gynostemma pentaphyllum (Thunb.) Makino (Chinese name, jiaogulan) is an oriental medicinal herb with numerous pharmacological activities, such as antitumor, cholesterol-lowering, immunopotentiating, antioxidant, hypoglycemic, and antidiabetic effects (Norberg et al. 2004). Phytochemical studies of $G$. pentaphyllum have identified approximately 90 dammarane-type saponin glycosides, known as gypenosides, which are responsible for its pharmacological activities (Yin et al. 2004). The chemical structure of gypenosides closely resembles that of ginsenosides found in Panax ginseng (Yin et al. 2004). Since ginsenosides are the well-known biologically active constituents in Korean ginseng, G. pentaphyllum has also received considerable attention. However, there is limited information on gypenoside production in plant cell cultures (Fei et al. 1993), probably because their numerous pharmacological activities have only recently been discovered (Norberg et al. 2004).

Hairy root cultures have been proposed as an alternative method of producing plant secondary metabolites because of they have genetic and biochemical stability, rapid growth rate and a capability to synthesize secondary products at levels comparable to that of the original plants (Sevón \& Oksman-Caldentey 2002). Although the initiation of $G$. pentaphyllum hairy roots has been described in the Chinese language (Fei et al. 1993), there are no data for systematic studies on gypenoside production by transformed root cultures. This work is concerned with the establishment of transformed root cultures of $G$. pentaphyllum and investigates their utility in gypenoside production. Variations in growth and gypenoside content of different root clones were examined to evaluate the effect of clonal selection in gypenoside accumulation. The effects of medium composition on growth and gypenoside accumulation of $G$. pentaphyllum hairy roots were also investigated.

\section{Materials and methods}

\section{Bacterium}

Agrobacterium rhizogenes ATCC 15834 was grown in $40 \mathrm{ml}$ YEB medium (Vervliet et al. 
1975) at $28^{\circ} \mathrm{C}$ with shaking at $100 \mathrm{rpm}$. When $\mathrm{OD}_{600}$ reached 1, the culture was centrifuged and the cells resuspended in $40 \mathrm{ml}$ hormone-free MS liquid medium (Murashige \& Skoog 1962), supplemented with $20 \mu \mathrm{m}$ acetosyringone.

\section{Establishment of hairy root clones}

Gynostemma pentaphyllum (Thunb.) Makino, a native plant of Taiwan, was obtained from the Herbal Plant Garden of the Taitung District Agricultural Research and Extension Station (Taiwan). Young leaves of G. pentaphyllum were sterilized with $70 \%(\mathrm{v} / \mathrm{v})$ ethanol for $30 \mathrm{~s}$ and $1 \%(\mathrm{w} / \mathrm{v}) \mathrm{NaOCl}$ for $5-10 \mathrm{~min}$, then rinsed four times with sterile distilled water. Hairy roots were induced from leaf discs by co-culturing with $A$. rhizogenes. The inoculated leaves were transferred to MS solid medium and kept at $25{ }^{\circ} \mathrm{C}$ in darkness. Hairy roots typically appeared at the infected sites 2 weeks following transfer. Single roots (15-20 $\mathrm{mm}$ in length) were excised and transferred to hormone-free MS solid medium supplemented with $300 \mathrm{mg}$ carbenicillin $1^{-1}$ for eliminating the bacteria. Following several passages of subculture (at 7-10 day intervals), the rapidly growing hairy roots without bacterial contamination were transferred to fresh medium at 4-week intervals.

\section{Identification of transformed hairy roots using PCR}

Genomic DNA was extracted and purified from both fresh transformed and native roots according to the method outlined by Thomson \& Henry (1995). Approx. 100 ng genomic DNA was used for PCR. The following primers were used to amplify the TL-DNA rolB sequences: 5'-GATGGGCTCTTGCAGT $-3^{\prime}$ and $5^{\prime}$-GGCTCCGGTGAGGAG- $3^{\prime}$. The primers $5^{\prime}$-ATCATTTGTAGCGACT- $3^{\prime}$ and $5^{\prime}$-AGCTCAAACCTGCTTC- ${ }^{\prime}$ were designed based on the virC gene from the not-transferred virulence region of the $A$. rhizogenes Ri plasmid. For amplification, initial denaturation was performed at $94{ }^{\circ} \mathrm{C}$ for $3 \mathrm{~min}$, followed by 35 cycles of amplification (each comprising $1 \mathrm{~min}$ at $94{ }^{\circ} \mathrm{C}, 1 \mathrm{~min}$ at $55^{\circ} \mathrm{C}$ and $1 \mathrm{~min}$ at $72{ }^{\circ} \mathrm{C}$ ) and a final extension at $72{ }^{\circ} \mathrm{C}$ for $5 \mathrm{~min}$. The amplified products were separated on a $1.5 \%(\mathrm{w} / \mathrm{v})$ agarose gel with $0.5 \mu \mathrm{g}$ ethidium bromide $\mathrm{ml}^{-1}$ and detected under UV light.

\section{Culture experiments for hairy roots}

The biomass and metabolite accumulation of various hairy root clones were assessed using MS and B5 media (Gamborg et al. 1968). Different salt strengths of MS liquid media were used to investigate the influence of basal medium salt strength on root growth and metabolite production in shake flasks. The medium $\mathrm{pH}$ was adjusted to 5.7. Nine root tips ( $30 \mathrm{~mm}$ in length) were inoculated into a $125-\mathrm{ml}$ flask containing $30 \mathrm{ml}$ hormone-free liquid medium with $30 \mathrm{~g}$ sucrose $1^{-1}$ and cultured on an orbital shaker at $80 \mathrm{rpm}$ in darkness at $25^{\circ} \mathrm{C}$ for 49 days. All experiments were performed in triplicate.

\section{Analyses}

Harvested hairy roots were washed twice using doubled-distilled water, then gently pressed onto filter papers to remove excess water and weighed. Dry weight was measured by drying the fresh hairy roots in an oven at $50{ }^{\circ} \mathrm{C}$ until constant weight was achieved.

To measure gypenosides, $50 \mathrm{mg}$ powdered dry hairy roots was extracted using $5 \mathrm{ml} 80 \%$ (v/v) methanol in an ultrasonic bath at $50{ }^{\circ} \mathrm{C}$ for $30 \mathrm{~min}$, then transferred to a shaker and kept at $4{ }^{\circ} \mathrm{C}$ for $24 \mathrm{~h}$ with shaking at $100 \mathrm{rpm}$. Following filtration, the extracts were evaporated using a vacuum pump and then redissolved in water. The water-soluble fraction was washed with ether and extracted with water-saturated $n$-butanol. The organic phase was evaporated until dry and redissolved in water. Purified gypenosides were obtained after further purification using Sep-Pak $\mathrm{C}_{18}$ and Sep-Pak aminopropyl solid-phase extraction cartridges according to the method described by Li et al. (1996).

Quantitative analysis of gypenosides was performed using the modified method described by Akalezi et al. (1999). First, $200 \mu \mathrm{l}$ purified saponin solution was heat evaporated, then mixed with $0.2 \mathrm{ml} 5 \%(\mathrm{w} / \mathrm{v})$ vanillin in glacial acetic acid and $0.8 \mathrm{ml}$ perchloric acid, then incubated for $15 \mathrm{~min}$ in a water bath at $60^{\circ} \mathrm{C}$. Following cooling, the sample was mixed with $5 \mathrm{ml}$ glacial acetic acid. The absorbancy of the mixture at $550 \mathrm{~nm}$ was then measured. Ginsenoside Rb1 was used as the standard. The average of two measurements for each of three different cultures was calculated. 


\section{Results and discussion}

\section{Establishment of hairy root cultures}

Hairy root cultures of $G$. pentaphyllum were initiated by inoculation of leaf discs with $A$. rhizogenes ATCC 15834. These roots exhibited characteristics typical of transformed roots, that is, rapid growth, extensive lateral branch and a lack of geotropism (Sevón \& Oksman-Caldentey 2002). The transformed root was confirmed by PCR to determine the presence of a T-DNA sequence in their genomes (Figure 1). The PCR products from the hairy roots for rolB regions but not from untransformed roots gave the expected $540 \mathrm{bp}$. This finding indicated that the $r o l \mathrm{~B}$ genes from the $\mathrm{Ri}$ plasmid of $A$. rhizogenes were integrated into the genome of $G$. pentaphyllum hairy roots. The negative results of PCR amplification for the virC gene demonstrated that no bacterial DNA was involved in $r o l \mathrm{~B}$ amplification leading to false positives.

Variation among $\mathrm{G}$. pentaphyllum hairy root clones

Root growth for the established hairy root clones of the two basal media differed significantly (Figure 2a). Additionally, gypenoside production was significantly different among the individual hairy root

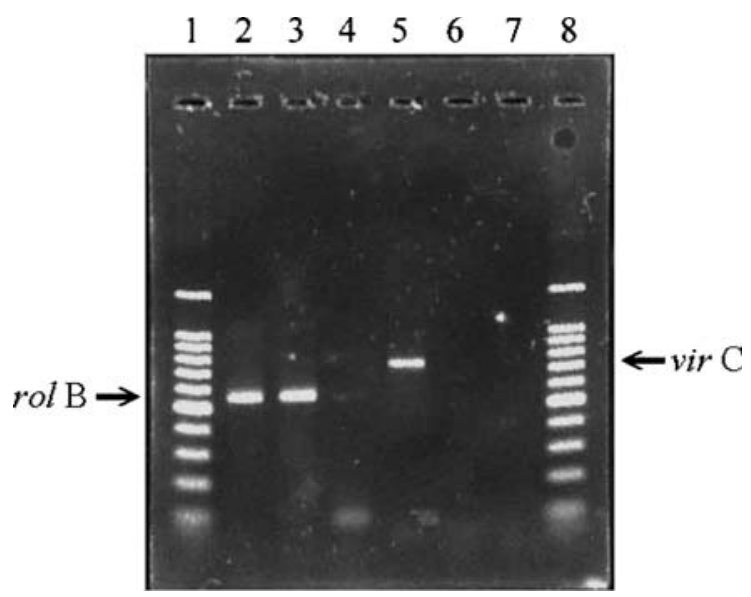

Fig. 1. PCR analysis of hairy roots. PCR analysis was performed using primers for the rooting locus genes TL-rolB (lanes 2-4). Control amplification was performed using virC primers (lanes 5-7). Lanes 1, 8: marker DNA (100 bp DNA ladder); lanes 2, 5: $A$. rhizogenes plasmid DNA; lanes 3, 6: $G$. pentaphyllum hairy roots genomic DNA; and lanes 4, 7: $G$. pentaphyllum genomic DNA. clones (Figure 2b). Taking into account both growth and the biomass of gypenoside concentrations, clone GP-01, which had the highest number of gypenosides and growth rate in both MS and B5 media, was selected for further experimentation.

\section{Effects of culture media}

When the root clone GP-01 was cultured in serially diluted MS media, the increase in growth of hairy roots was positively correlated with the concentration of inorganic salts, and no significant difference was observed in the gypenoside content of roots with different MS salt strengths (Table 1). Cultured media test results indicated that the full-strength MS medium was superior to the other media for both root growth and total gypenoside production.

Time course of $\mathrm{G}$. pentaphyllum hairy root cultures

Following 49 days of cultivation in MS medium the largest dry weight of $G$. pentaphyllum hairy roots increased about 120 -fold compared to inoculum, and the gypenoside content of $38 \mathrm{mg} \mathrm{g}^{-1}$ dry wt was achieved (Figure 3a). Gypenoside production increased with biomass until day 49, with a peak value of approximately $280 \mathrm{mg}^{-1}$ (Figure $3 \mathrm{a}$ ). Both the dried root samples and culture media were analyzed for gypenoside content. The medium was free of gypenosides throughout the cultivation process.

Kinetics study revealed that accumulation of gypenosides in $G$. pentaphyllum hairy roots was "growth-associated" (Figure 3b), where the concentration of product per unit weight remained constant during the growth cycle (Wilson et al. 1987). The result in this study closely resembles that for hairy roots of the Solanum aviculare (Subroto \& Doran 1994) and Catharanthus roseus (Bhadra \& Shanks 1997), indicating that the biosynthesis of gypenosides in $G$. pentaphyllum hairy roots occurred simultaneously with the biosynthesis of other primary metabolites used for growth. This pattern differs from the production behavior of most secondary metabolites, which showing that significant levels of production are observed after growth ceases (Payne et al. 1992).

The gypenoside content of hairy roots cultured in MS medium for 49 days was compared 

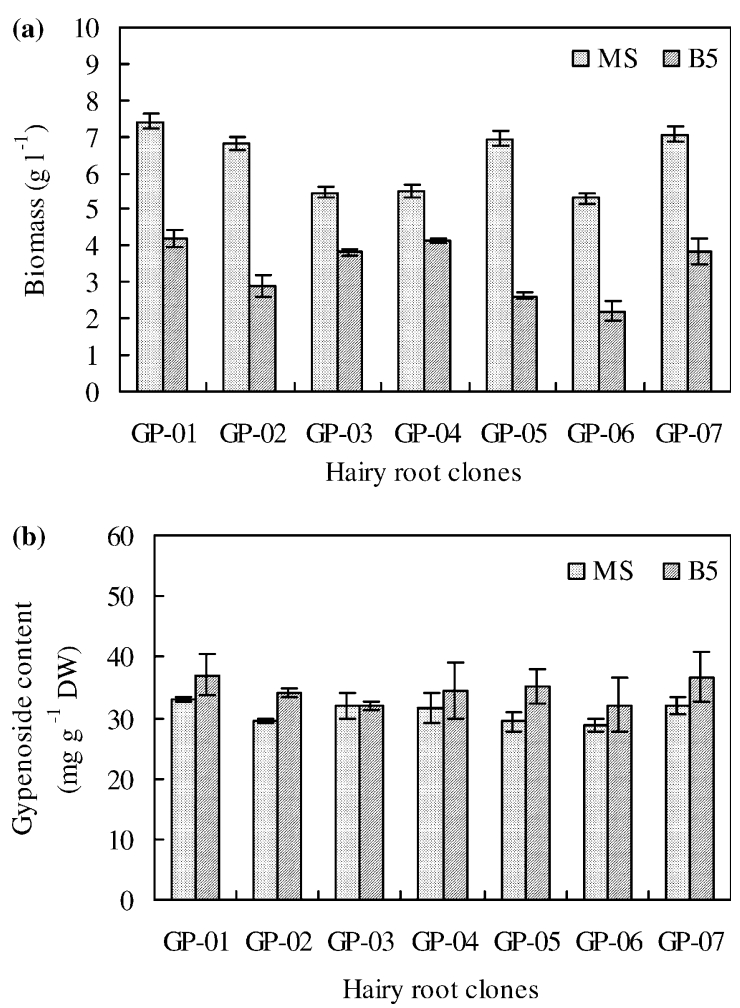

Fig. 2. (a) Growth; and (b) gypenoside content of G. pentaphyllum hairy root clones. Seven hairy root clones were cultured for 49 days in either MS or B5 liquid medium without growth regulators. Values are means of triplicate results with standard deviations.

with those of various origins of G. pentaphyllum (Table 2). In this work, gypenoside content of $38 \mathrm{mg} \mathrm{g}^{-1}$ dry wt was obtained using $G$. penta-

Table 1. Effects of different salt strengths in MS media on root growth and gypenoside production of $G$. pentaphyllum transformed root cultures.

\begin{tabular}{llll}
\hline Material & Medium & $\begin{array}{l}\text { Dry } \\
\text { weight } \\
\left(\mathrm{g} \mathrm{l}^{-1}\right)\end{array}$ & $\begin{array}{l}\text { Gypenoside } \\
\text { content } \\
\left(\mathrm{mg} \mathrm{g}^{-1} \text { dry wt }\right)\end{array}$ \\
\hline Hairy roots & $1 / 8 \mathrm{MS}$ & $1.5 \pm 0.1$ & $30 \pm 2.6$ \\
& $1 / 4 \mathrm{MS}$ & $4.1 \pm 0.7$ & $29 \pm 0.9$ \\
& $1 / 2 \mathrm{MS}$ & $5.9 \pm 0.1$ & $27 \pm 2.2$ \\
& $\mathrm{MS}$ & $7.3 \pm 0.1$ & $33 \pm 0.8$ \\
& $3 / 2 \mathrm{MS}$ & $7.0 \pm 0.4$ & $34 \pm 2.4$
\end{tabular}

Hairy roots were cultivated in 125 -ml shake flasks containing $30 \mathrm{ml}$ of hormone-free liquid basal media. Biomass and gypenoside productivity were measured after 7 weeks of cultivation. Values are means of triplicate results with standard deviations. phyllum hairy root cultures, which was significantly higher than that previously reported for hairy root cultures (Fei et al. 1993), and was comparable with value in the leaves of the parent plant (Table 2). In this study, high levels of gypenosides were obtained from hairy roots despite distribution results showing relatively low gypenoside levels in native roots of $G$. pentaphyllum (Liu et al. 2005). This study demonstrated that transformed roots can synthesize and store significant quantities of secondary metabolites (Table 2).

Although the hairy roots under these conditions produced approximately $30 \%$ to $40 \%$ less gypenosides than commercial sources of $G$. pentaphyllum (Table 2), the growing time was much shorter when compared to field-grown plants. With hairy root cultures, product quality and quantity are easy to control because natural variances in seasonal climates and geographical environments are excluded and culture conditions and process variables are easily optimized (Payne et al. 1992). The hairy root cultures have been considered as a potential alternative for production of gypenosides. Several strategies for the enhancement of biomass and gypenosides have been adopted in which the effects of medium compositions, culture conditions and elicitations

Table 2. Gypenoside contents from various origins of G. pentaphyllum and hairy roots.

\begin{tabular}{ll}
\hline Material & $\begin{array}{l}\text { Gypenoside content } \\
\left(\mathrm{mg} \mathrm{g}^{-1} \text { dry wt) }\right.\end{array}$ \\
\hline Commercial product No.1 & $57 \pm 1.3$ \\
Commercial product No.2 & $60 \pm 1.8$ \\
Commercial product No.3 & $61 \pm 3.4$ \\
Commercial product No.4 & $53 \pm 1.7$ \\
Commercial product No.5 & $66 \pm 1.2$ \\
Leaves of parent plant & $42 \pm 1.8$ \\
Roots of parent plant & $15 \pm 2.4$ \\
Hairy roots & $38 \pm 1.4$ \\
\hline
\end{tabular}

Commercial products (packaging as tea bags) were leaves of the field-grown G. pentaphyllum derived from China and Taiwan. The parent plant used for induction of G. pentaphyllum hairy roots was obtained from the Herbal Plant Garden of the Taitung District Agricultural Research and Extension Station (Taiwan). Hairy roots were cultivated in 125 -ml shake flasks containing $30 \mathrm{ml}$ of hormone-free MS liquid media for 49 days. Values are means of triplicate results with standard deviations. 

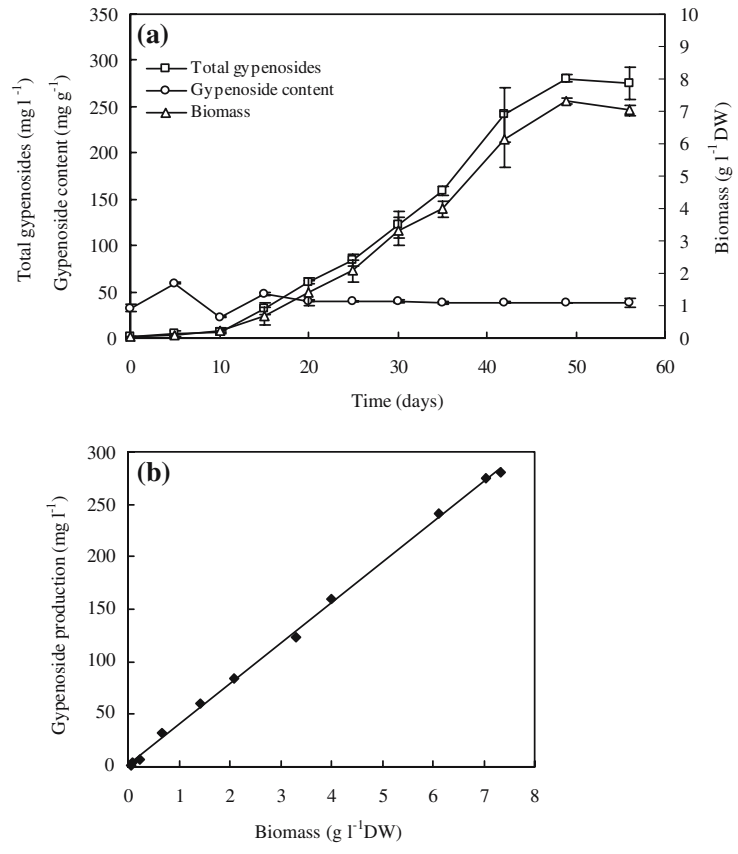

Fig. 3. (a) Time course; and (b) correlation of total gypenosides with dry biomass in shake-flask cultures of G. pentaphyllum hairy roots. The hairy root cultures were grown in $125-\mathrm{ml}$ flasks containing $30 \mathrm{ml}$ of MS liquid media. Every 5-7 days, triplicate flasks were harvested for measurement of biomass and gypenoside productivity. Values are means of triplicate results with standard deviations.

on root growth and gypenoside accumulation are currently under research.

\section{Acknowledgments}

Dr. Yu-Tien Liu (National Defense Medical Center, Taiwan) and Dr. Kung-Ta Lee (National Taiwan University, Taiwan) are appreciated for helpful opinion and discussion. We also thank Mr. Sing-Jin Lee (Taitung District Agricultural Research and Extension Station, Taiwan) for kindly providing the G. pentaphyllum plants.

\section{References}

Akalezi CO, Liu S, Li QS, Yu JT, Zhong JJ (1999) Combined effects of initial sucrose concentration and inoculum size on cell growth and ginseng saponin production by suspension cultures of Panax ginseng. Process Biochem. 34: 639-642.

Bhadra R, Shanks JV (1997) Transient studies of nutrient uptake, growth, and indole alkaloid accumulation in heterotrophic cultures of hairy roots of Catharanthus roseus. Biotechnol. Bioeng. 55: 527-534.

Fei HM, Mei KF, Shen X, Ye YM, Lin ZP, Peng LH (1993) Transformation of Gynostemma pentaphyllum by Agrobacterium rhizogenes and saponin production in hairy root cultures. Acta Bot. Sin. 35: 626-631.

Gamborg OL, Miller RA, Ojima K (1968) Nutrient requirements of suspension cultures of soybean root cells. Exp. Cell Res. 50: 151-158.

Li TSC, Mazza G, Cottrell AC, Gao L (1996) Ginsenosides in roots and leaves of American ginseng. J. Agric. Food Chem. 44: 717-720.

Liu SB, Lin R, Hu ZH (2005) Histochemical localization of ginsenosides in Gynostemma pentaphyllum and the content changes of total gypenosides. Acta Biol. Exp. Sin. 38: 54-60.

Murashige T, Skoog F (1962) A revised medium for rapid growth and bioassays with tobacco tissue cultures. Physiol. Plant 15: 473-497.

Norberg A, Hoa NK, Liepinsh E, Phan DV, Thuan ND, Jörnvall H, Sillard R, Östenson C-G (2004) A novel insulinreleasing substance, phanoside, from the plant Gynostemma pentaphyllum. J. Biol. Chem. 279: 41361-41367.

Payne GF, Bringi V, Prince C, Shuler ML (1992) Plant Cell and Tissue Culture in Liquid Systems. New York: Hanser Publishing.

Sevón N, Oksman-Caldentey K-M (2002) Agrobacterium rhizogenes-mediated transformation: Root cultures as a source of alkaloids. Planta Med. 68: 859-868.

Subroto MA, Doran PM (1994) Production of steroidal alkaloids by hairy roots of Solanum aviculare and the effect of gibberellic acid. Plant Cell Tiss. Org. Cult. 38: 93-102.

Thomson D, Henry R (1995) Single-step protocol for preparation of plant tissue for analysis by PCR. BioTechniques 19: 394-400.

Vervliet G, Holsters M, Teuchy H, van Montagu M, Schell J (1975) Characterization of different plaque forming and defective temperate phages in Agrobacterium strains. J. Gen. Virol. 26: 33-48.

Wilson PDG, Hilton MG, Robins RJ, Rhodes MJC (1987) Fermentation studies of transformed root cultures. In: Moody GW, Baker PB, eds. Bioreactors and Biotransformation, London: Elsevier Press, pp. 38-51.

Yin F, Hu LH, Pan RX (2004) Novel dammarane-type glycosides from Gynostemma pentaphyllum. Chem. Pharm. Bull. 52: 1440-1444. 